\title{
Parasites and parasite management practices of organic and conventional dairy herds in Minnesota
}

\author{
U. S. Sorge, ${ }^{11}$ R. D. Moon, † B. E. Stromberg, $¥$ S. L. Schroth, ${ }^{*}$ L. Michels, ${ }^{*}$ L. J. Wolff, ${ }^{*}$ D. F. Kelton,\# \\ and B. J. Heins§ \\ *Department of Veterinary Population Medicine, \\ †Department of Entomology, and \\ fDepartment of Veterinary and Biomedical Sciences, University of Minnesota, St. Paul 55108 \\ §Department of Population Medicine, University of Guelph, Guelph, ON, N1G 2W1, Canada \\ \#Department of Animal Science, University of Minnesota, St. Paul 55108
}

\section{ABSTRACT}

The objective of this study was to describe the prevalence and practices used to manage internal helminth parasites and external arthropod parasites on organic and conventional dairy herds in Minnesota. All organic (ORG) dairy herds in Minnesota $(\mathrm{n}=114)$ and a convenience sample of conventional herds were invited to participate in the study. Thirty-five ORG herds and 28 conventional herds were visited once in summer and fall of 2012. Conventional dairy herds were split into small conventional (SC, < 200 cows) and medium-sized conventional herds ( $\mathrm{MC}, \geq 200$ cows) so that SC herds were comparable in size to the ORG herds. Dairy managers were surveyed to assess their farm management practices and perceptions about parasites, hygiene scores were recorded for adult stock, and fecal samples were collected from a nominal 20 breeding-age heifers to characterize abundance of internal parasites. Nonparametric tests were used to compare fecal egg counts per gram (FEC) among farms grouped by management systems and practices. Organic farms had more designated pasture and were more likely to use rotational grazing compared with conventional farms, but the stocking densities of animals on pasture were similar among farm types. The overall FEC were very low, and only a few individual ORG heifers had FEC >500 eggs/ gram. Samples from heifers on ORG farms had significantly more strongyle-type eggs than those on SC and MC farms (ORG: $6.6 \pm 2.1$; SC: $0.5 \pm 0.3$; MC: $0.8 \pm$ 0.7 ), but egg counts of other types of gastrointestinal parasites did not differ significantly among the 3 herd groups. Fly control measures were applied mainly to milking cows and preweaned calves and were used on $88.6 \%$ of ORG herds, $60.0 \%$ of SC herds, and $91.7 \%$ of $\mathrm{MC}$ herds. Approximately half of the producers re-

Received October 28, 2014.

Accepted January 12, 2015.

${ }^{1}$ Corresponding author: sorge@umn.edu ported having seen skin conditions suggestive of lice or tail mange in their cattle during the previous winter (ORG: $48.6 \%$, SC: $57.1 \%$, MC: 53.9\%). Although most conventional producers reported treating these skin conditions, most organic producers stated they had not treated them. In conclusion, gastrointestinal parasite egg counts were low overall at the time of the survey, and most surveyed producers did not perceive gastrointestinal parasites to be a problem for their animals' health. Independent of the herd type, fly control was mostly targeted at the lactating herd and preweaned calves.

Key words: dairy cattle, gastrointestinal parasite, flies, ectoparasite, organic

\section{INTRODUCTION}

During the second half of the 20th century, many dairy farmers moved away from pasture-based housing systems and housed their dairy cows indoors year round. This shift in housing system decreased exposure of cattle to pasture-contaminating gastrointestinal (GI) parasites. In addition, development and use of pharmaceutical parasiticides decreased the likelihood of heavy parasite infestations and reduced related production losses (Fox et al., 2007).

In contrast, organic (ORG) dairying has increased in the United States over the past decade. Between 2000 and 2008, the number of organically managed cows in the United States increased 6-fold, from 38,196 to 249,766 dairy cows (ERS, 2013). The Agricultural Marketing Service of the USDA oversees the National Organic Program (NOP) and specifies requirements for ORG production (USDA-AMS, 2012). The NOP rules prohibit the use of hormones and antibiotics, including ionophores, and require that ruminants over 6 mo of age receive at least $30 \%$ of their DMI from pasture during a minimum grazing season of $120 \mathrm{~d}$ per year. The NOP also specifically states that a producer must not withhold effective treatment (e.g., antimicrobials) solely to 
maintain the organic status of the animal. If an animal requires treatment that is not explicitly allowed under NOP regulations, then the animal has to be removed from the farm in a timely manner and its milk or meat can never be sold as organic. Furthermore, parasiticides must never be used on a routine basis, but 3 substances may be used as emergency treatments; that is, if preventive management practices fail. In that instance, the organic certifier has to approve the use of these parasiticides for that particular farm before they can be used. If they are used without the certifier's approval, the animals and farm will lose their organic status. Ivermectin has been on the list of approved dewormers since the original rules were adopted, and fenbendazole and moxidectin were added in 2012. The 3 substances are allowed for use in dairy and breeding stock, but are not allowed for slaughter stock, during lactation, or in the last trimester of gestation in breeding stock.

Compared with conventional herd management practices, the pasture requirement and limited use of parasiticides specified in the NOP rules have the potential to increase the prevalence of GI parasites in organically managed dairy herds. Given a general lack of knowledge about the prevalence of internal and external parasites among dairy herds in the upper Midwest, we conducted the present study to describe and compare the management practices and occurrence of internal helminth parasites and external arthropod parasites on conventional and ORG dairy farms in Minnesota. We hypothesized that ORG herds would have higher fecal egg counts than conventional herds.

\section{MATERIALS AND METHODS}

In spring 2012, all ORG dairy farms $(\mathrm{n}=114)$ in Minnesota were invited to participate in the study. In addition, a convenience sample of neighboring conventional farms was enrolled for comparison. The conventional farms participated in DHIA, raised their own replacement heifers, and housed cows either on pasture or in confinement.

\section{Farm Characteristics}

Enrolled farms were visited once in 2012 to administer a questionnaire about general herd management practices, types of animal housing, frequency of disease events, perceptions of disease importance, and treatments used for internal and external parasite control. The questionnaire was largely based on 3 different National Animal Health Monitoring questionnaires from the Dairy 2007 study (General Dairy Management Report, Veterinary Services Initial Survey, and Veterinary Services Second Visit). The study and survey protocol were approved by the University of Minnesota's Institutional Animal Care and Use Committee (protocol \#1111B06824). A copy of the full survey instrument can be obtained from the corresponding author upon request. Results concerning parasites and parasite management will be summarized in the present report, and further aspects of animal housing and frequency of disease events will be summarized elsewhere.

\section{Fecal Samples and Hygiene Scores}

At time of visit, fecal samples were collected rectally or from freshly passed dung from a nominal 20 breeding-age heifers per farm (Gasbarre et al., 1996). Gastrointestinal parasites were extracted using the Wisconsin sugar float method (Bliss and Kvasnicka, 1997). Samples were scored for presence of coccidial oocysts. Eggs of helminth nematodes, cestodes, and trematodes were identified to family or genus and counted. Additionally, mature cows, both dry and milking, or approximately $20 \%$ thereof, were examined during the farm visits and rated for cleanliness, using a hygiene scoring system with scale of 1 for clean to 4 for extremely dirty (FARM, 2013).

\section{Statistical Analysis}

Data at the herd level were analyzed in SAS 9.4 (SAS Institute Inc., Cary, NC). Dairy farms were grouped for comparison into 3 types: ORG, small conventional herds (SC, $<200$ mature cows) and medium-sized conventional herds (MC, $\geq 200$ mature cows). Mature cows included both dry and milking cows, and conventional herds were split by size so that SC herds were similar in size to the ORG herds. Furthermore, herds with strip, mob, and rotational grazing were combined as rotational grazing and compared with herds that used conventional grazing or housed cattle indoors. Categorical variables were summarized using frequency statistics (PROC FREQ). Fecal egg counts among heifers at each of 60 sampled farms were examined graphically with box-whisker plots ranked by medians within and among herd types, using the boxplot procedure in R (R Core Team 2014). Average fecal egg counts [FEC in eggs/g of feces (epg)] were calculated as geometric mean for heifers in each herd, and a Kruskal-Wallis test was used to compare median geometric mean among herds in the 3 dairy types, followed by Mann-Whitney U-tests for pairwise comparisons (e.g., Tables 1 and 3). Associations among herd types and categorical variables were assessed with Fisher's exact tests, first across all 3 herd types, and then followed by pairwise comparisons if differences $(P<0.1)$ were initially found among herd types (e.g., Tables 1, 2, 4, and 5). Spearman correlation 
Table 1. Median values (25th-75th quartiles in parentheses) for characteristics or percentages of 63 Minnesota dairies on day of visit in June to November 2012

\begin{tabular}{|c|c|c|c|c|}
\hline \multirow[b]{2}{*}{ Characteristic } & \multicolumn{3}{|c|}{ Herd type $^{1}$} & \multirow[b]{2}{*}{$P$-value ${ }^{2}$} \\
\hline & ORG & $\mathrm{SC}$ & $\mathrm{MC}$ & \\
\hline Milking cows (no.) & $68(42-110)^{\mathrm{b}}$ & $57(50-84)^{\mathrm{b}}$ & $285(195-383)^{\mathrm{a}}$ & $<0.01$ \\
\hline Rolling herd average (kg) & $\begin{array}{l}6,363^{\mathrm{b}} \\
(5,522-7,272)\end{array}$ & $\begin{array}{l}10,363^{\mathrm{a}} \\
(8,572-11,876)\end{array}$ & $\begin{array}{l}11,363^{\mathrm{a}} \\
(10,681-11,710)\end{array}$ & $<0.01$ \\
\hline Most current BTSCC ${ }^{3}\left(10^{3} \times\right.$ cells $\left./ \mathrm{mL}\right)$ & $300(225-330)^{\mathrm{a}}$ & $226(162-282)^{\mathrm{b}}$ & $220(173-240)^{\mathrm{b}}$ & 0.03 \\
\hline \multicolumn{5}{|l|}{ Access to pasture (\% of herds) } \\
\hline Lactating herd & $100^{\mathrm{a}}$ & $46.7^{\mathrm{b}}$ & $7.7^{\mathrm{c}}$ & $<0.01$ \\
\hline Dry cows & $97.1^{\mathrm{a}}$ & $73.3^{\mathrm{b}}$ & $30.8^{\mathrm{c}}$ & $<0.01$ \\
\hline Weaned heifers & $100^{\mathrm{a}}$ & $80.0^{\mathrm{a}}$ & $38.5^{\mathrm{b}}$ & $<0.01$ \\
\hline Pasture management (\% of herds with pasture access) & & & & $<0.01$ \\
\hline Rotational & $100^{\mathrm{a}}$ & $23.1^{\mathrm{b}}$ & $0^{\mathrm{b}}$ & \\
\hline Continuous & 0 & 76.9 & 100 & \\
\hline \multicolumn{5}{|l|}{ Pasture stocking density (cows/ha) } \\
\hline
\end{tabular}

${ }^{\mathrm{a}-\mathrm{c}}$ Values in the same row with different superscripts were significantly different with pairwise Fisher's exact tests $(\alpha=0.05)$.

${ }^{1}$ ORG $=$ organic herds, $\mathrm{SC}=$ small conventional herds $(<200$ dry and milking cows $)$, and $\mathrm{MC}=$ medium-sized conventional herds $(\geq 200$ cows $)$. $P$-values are for differences in medians among the 3 herd types, based on Kruskal-Wallis tests $(\alpha=0.05)$. Medians in the same row with different superscripts judged significantly different by pairwise Mann-Whitney U-test comparisons $(\alpha=0.05)$.

${ }^{2} P$-values for differences in overall percentages among the 3 herd types based on Fisher's exact test $(\alpha=0.05)$.

${ }^{3}$ Bulk tank SCC.

coefficients were used to assess associations between continuous variables (PROC CORR Spearman). The significance level was set at $\alpha=0.05$.

\section{RESULTS}

\section{Farm Characteristics}

Thirty-three ORG herds, $15 \mathrm{SC}$ herds, and $13 \mathrm{MC}$ herds were visited between June and September 2012. Median visit dates among the 3 herd types were July 18, August 22, and August 12, respectively. Two remaining ORG herds were visited in October and November. The removal of these 2 late ORG herds from analysis did not affect the results, so data from those 2 herds were retained in the analyses. Characteristics of the 63 study farms are summarized in Table 1. Organic dairy herds produced, on average, significantly less milk per cow and had a higher producer-reported recent bulk tank SCC than SC or MC herds. Proportions of cows that were dirty (hygiene score $\geq 3$ ) were not associated with any particular herd type $(P=0.56)$. Organic herds had pasture access for almost all animals (Table 1 ). Likewise, many SC herds allowed their cows (lactating cows: $\mathrm{n}=7$; dry cows: $\mathrm{n}=11$ ) and heifers $(\mathrm{n}=$ 12) on pasture during the summer months. In contrast, most MC dairies housed weaned and breeding-age heifers in indoor group pens, and housed lactating cows in freestall barns. However, some MC dairies allowed their dry $(\mathrm{n}=4)$ or lactating cows $(\mathrm{n}=1)$ as well as their heifers $(\mathrm{n}=5)$ access to pasture.

Patterns of pasture management also differed among the 3 dairy types that used summer pasture (Table 1). Organic dairies had more designated pasture acreage than $\mathrm{SC}$ or $\mathrm{MC}$ dairies that used pasture. All ORG dairies used some form of rotational grazing, whereas most of the SC dairies and all of the MC dairies with pasture used continuous grazing (Table 1). Most commonly, replacement heifers did not have access to the same pastures as cows (ORG: $51.5 \%$, SC: $45.4 \%$, MC: $61.0 \%)$, but some producers grazed heifers with cows (ORG: $27.3 \%$, SC: $45.5 \%$, MC: $40.0 \%$ ) or after cows (ORG: $21.2 \%$, SC: $9.1 \%$, MC: 0\%). Although the average stocking densities for mature cows and replacement heifers were numerically different, densities did not differ among dairy types $(P \geq 0.25)$. We detected no significant difference in the date that cows and heifers had been turned out to pasture $(P>0.1)$.

Producer responses to survey questions concerning perceptions and management of GI and external parasites will be summarized in corresponding sections below.

\section{GI Parasites}

Producers generally thought that heifers were the most affected by GI parasites, but only 6 ORG managers (17.1\%), 1 SC manager (11\%), and none of the MC 
Table 2. Percentages of farms with gastrointestinal parasites of different types detected in heifer fecal samples, by dairy type

\begin{tabular}{lccccc}
\hline & \multicolumn{3}{c}{ Herd type $^{1}$} & & \\
\cline { 2 - 4 } Gastrointestinal & ORG & SC & MC & Overall & $P_{\text {-value }}^{2}$ \\
\hline parasite eggs & 94 & 100 & 100 & 97 & $>0.99$ \\
Eimeria & $100^{\mathrm{a}}$ & $80^{\mathrm{b}}$ & $67^{\mathrm{b}}$ & 88 & $<0.01$ \\
Strongyle & $52^{\mathrm{b}}$ & $67^{\mathrm{b}}$ & $92^{\mathrm{a}}$ & 63 & 0.04 \\
Trichuris & 42 & 67 & 50 & 50 & 0.28 \\
Nematodirus & $68^{\mathrm{a}}$ & $7^{\mathrm{b}}$ & $8^{\mathrm{b}}$ & 42 & $>0.01$ \\
Moniezia & 16 & 13 & 17 & 15 & $>0.99$ \\
Strongyloides & 16 & 7 & 8 & 12 & 0.66 \\
Capillaria & 19 & 0 & 0 & 10 & 0.08 \\
Dictyocaulus & 7 & 7 & 0 & 5 & $>0.99$ \\
Buxtonella & 7 & 7 & & 5
\end{tabular}

${ }^{a, b}$ Percentages in the same row with different superscripts were significantly different by pairwise Fisher's exact tests $(\alpha=0.05)$.

${ }^{1} \mathrm{ORG}=$ organic herds $(\mathrm{n}=33), \mathrm{SC}=$ small conventional herds $(<200$ cows, $\mathrm{n}=15), \mathrm{MC}=$ medium-sized conventional herds $(\geq 200$ mature cows, $\mathrm{n}=12)$.

${ }^{2} P$-values are for differences in overall percentages among the 3 herd types, based on Fisher's exact test $(\alpha=$ $0.05)$.

managers considered parasite prevention when setting up their grazing schedules for heifers $(P>0.99)$. Ultimately, GI parasites were only perceived as a problem by 7 ORG producers $(20.0 \%)$, whereas none of the SC or MC dairy herds thought the same $(P=0.08)$. The average FEC of those 7 ORG herds was significantly higher than averages of all other herds, including conventional and other ORG herds $(P=0.01)$ but did not differ statistically from the other ORG herds $(P>$ $0.34)$. Two ORG producers had used ivermectin in the previous year, which included only 1 of the 7 herds that saw GI parasites as problems for their herd. Less than one-third of the ORG producers $(22.9 \%)$ were aware that 2 pharmaceutical dewormers had been added to the NOP regulations in 2012. In contrast, $71.4 \%$ of SC and $92.3 \%$ of $\mathrm{MC}$ dairy herds regularly dewormed their cows or heifers (46.7 and $84.6 \%$, respectively) with pharmaceutical dewormers $(P<0.01)$.

A total of 1,083 fecal samples were obtained from replacement heifers of breeding age at 60 of the 63 enrolled dairies. Heifers at 2 ORG dairies and $1 \mathrm{MC}$ dairy were unavailable because they were housed at facilities separate from the main farms. Median sample size at the remaining dairies was 20 heifers (range 2-21); sample sizes at 7 ORG dairies and $3 \mathrm{SC}$ dairies were $<16$ animals, because fewer animals were available.

Oocysts of Eimeria and eggs of 8 other gastrointestinal parasites were detected in samples from the 60 dairies (Table 2). Eimeria were present on all but 2 ORG farms and on all of the conventional farms. The 2 ORG herds that were negative for Eimeria had sample sizes of $n=20$, and both herds used sawdust or wood shavings as bedding materials for their preweaned heifers but had no other management commonalities.
The remaining gastrointestinal parasites were less uniformly distributed among farms. Most of the dairies had 2 or more types of parasites, but 1 ORG dairy yielded only strongyle-type eggs (Strongylidae), and 5 conventional dairies yielded only Trichuris. Herdlevel prevalence of some of the parasites varied among dairy types (Table 2). Strongyles, the second most frequently encountered parasite after coccidia, were more prevalent on ORG dairies than on $\mathrm{SC}$ and $\mathrm{MC}$ dairies. Trichuris, the third most frequent parasite egg, was less prevalent on ORG and $\mathrm{SC}$ dairies than on MC dairies. Of the remaining parasites, Moniezia and Dictyocaulus were more prevalent on ORG dairies than on SC and MC dairies, whereas the prevalence of Nematodirus, Strongyloides, Capillaria, and Buxtonella did not differ significantly among the 3 types of dairies.

Within individual heifers, FEC (in epg) of many of the GI parasites were positively associated. Strongyle FEC were correlated with those of Strongyloides, Moniezia, Capillaria, and Nematodirus ( $\mathrm{r}>0.08 ; P<$ 0.01). Lungworm Dictyocaulus FEC were correlated with counts of the pasture-associated Moniezia ( $\mathrm{r}=$ 0.07; $P=0.03$ ), whereas counts of Nematodirus were positively correlated with counts of Strongyloides, Moniezia, and Trichuris $(\mathrm{r}>0.05 ; P<0.08)$. An exception occurred with Buxtonella, whose counts were independent of those of all the other gastrointestinal parasites $(P>0.15)$.

Heifer-level prevalence of Eimeria was almost $100 \%$ for heifers on Eimeria-positive farms, but Eimeria eggs were found in fewer MC heifers $[89 \%$, interquartile range (IQR): $75-95 \%$ ] than ORG (100\%, IQR: $95-100 \%)$ or SC heifers (100\%, IQR: $92-100 \% ; P<0.01)$. Infection rates for the remaining parasites were relatively lower 
Table 3. Median percentages (and 25th-75th quartiles) of heifers with detectable numbers of gastrointestinal parasite eggs in fecal samples, by parasite and dairy type

\begin{tabular}{lcccc}
\hline & \multicolumn{3}{c}{ Herd type $^{1}$} \\
Gastrointestinal \\
\cline { 2 - 4 } parasite eggs & ORG & SC & MC & $P$-value \\
\hline Strongyle & $100(93-100)^{\mathrm{a}}$ & $69(6-95)^{\mathrm{b}}$ & $18(0-29)^{\mathrm{c}}$ & $<0.01$ \\
Trichuris & $5(0-7)^{\mathrm{b}}$ & $5(0-10)^{\mathrm{b}}$ & $13(5-30)^{\mathrm{a}}$ & $<0.01$ \\
Nematodirus & $0(0-15)$ & $6(0-23)$ & $3(0-10)$ & 0.44 \\
Moniezia & $7(0-20)^{\mathrm{a}}$ & $0^{\mathrm{b}}$ & $0^{\mathrm{b}}$ & $<0.01$ \\
Strongyloides & 0 & 0 & 0 & 0.97 \\
Capillaria & 0 & 0 & 0 & 0.57 \\
Dictyocaulus & 0 & 0 & 0 & $>0.99$ \\
Buxtonella & 0 & 0 & 0 & 0.68 \\
\hline
\end{tabular}

${ }^{a-c}$ Frequencies in the same row with different superscripts were significantly different by Mann-Whitney U tests $(\alpha=0.05)$.

${ }^{1} \mathrm{ORG}=$ organic herds $(\mathrm{n}=33), \mathrm{SC}=$ small conventional herds $(<200$ dry and milking, $\mathrm{n}=15)$, and $\mathrm{MC}=$ medium-sized conventional herds ( $\geq 200$ mature cows, $\mathrm{n}=12$ ).

${ }^{2} P$-values are for overall differences in median percentages among the 3 herd types, based on Kruskal-Wallis tests $(\alpha=0.05)$.

and in parallel with herd-level prevalence (Table 3). Geometric mean FEC $( \pm$ SEM) of all helminth parasites combined were $7.9 \pm 2.3$ for ORG dairies, $0.5 \pm$ 0.3 for SC dairies, and $0.9 \pm 0.6$ for MC dairies, and the difference between ORG dairies and conventional dairies was significant $(P<0.01)$.

Strongyle eggs constituted the vast majority of all eggs; strongyle FEC were $6.6 \pm 2.1 \mathrm{epg}$ at ORG dairies, $0.5 \pm 0.3$ at $\mathrm{SC}$ dairies, and $0.8 \pm 0.7$ at $\mathrm{MC}$ dairies $(P<0.01$; Figure 1$)$. Most of the strongyle FEC from individual heifers were generally low, but 5 of the ORG herds had 1 or 2 heifers with FEC $>500$ epg. Herds that used pharmaceutical dewormers $(P=0.03)$, coccidiostats in feed $(P=0.08)$, or ionophores in feed $(P$ $<0.01$ ) had lower strongyle FEC than those that did not use them.

Interestingly, average Trichuris FEC were lower in ORG farms than in $\mathrm{MC}$ and nonpasture farms $(P=$ 0.04), whereas average Moniezia FEC were higher in ORG and pasture-access farms than in farms that continuously housed animals indoors $(P<0.01)$. Dictyocaulus eggs were found only in 6 ORG dairy herds $(P=0.02)$.

The FEC were also analyzed in relation to herd, pasture, and manure management practices (Table 4). Heifers on dairies that used rotational grazing had higher FEC than those that did not, and the longer the pasture regrowth time between consecutive grazings, the higher the FEC $(\mathrm{r}=0.48 ; P<0.01)$. However, this association vanished when the association was evaluated for ORG herds alone $(\mathrm{r}=0.28 ; P=0.11)$. Independent of management system, dairies that had a stream or pond on pasture had higher FEC than those that did not $(P<0.01)$, whereas dairies that used any kind of indoor feed line (e.g., post-and-rail) for weaned heifers had lower FEC $(P=0.02)$.

The FEC in heifers tended to be lower on farms where bedding for weaned heifers $(\mathrm{r}=-0.49 ; P<0.01)$ and for dry cows was changed more often $(\mathrm{r}=-0.51 ; P<$ 0.01 ). However, this relationship was not observed with bedding management for preweaned heifers $(P>0.22)$ or lactating cows $(P=0.67)$. Likewise, no relationship was observed between the percentage of cows with a hygiene score $\geq 3$ on farm, as a surrogate for animal hygiene on farm, and the FEC in heifers.

The FEC were independent of whether cows and heifers grazed in the same pastures, either at the same or different times $(P=0.77)$, and FEC were independent of stocking density on pasture $(P>0.63)$. Although the FEC tended to be higher when manure was spread over crops $(P=0.08)$, they were generally independent of whether manure was spread on pasture or on cropland $(P>0.28)$. The FEC were significantly lower on conventional farms that used pharmaceutical dewormers $(P$ $=0.02)$, coccidiostats $(P=0.06)$, or ionophores in feed $(P<0.01)$. Average FEC in heifers were significantly higher in the 7 ORG herds where managers thought GI parasites were problems than on all other farms $(P=$ 0.01). However, the average FEC of those 7 farms were not different from the other ORG farms $(P=0.18)$.

\section{Fly Control Measures}

Overall, a higher percentage of ORG and MC herds used some form of fly control compared with SC herds $(P=0.04)$. Table 5 shows the distribution of farms that used fly control on various animal groups and the fly control measures used by farms. 
Table 4. Percentages of herds diagnosed with different gastrointestinal parasites, grouped by access to pasture for heifers and management style

\begin{tabular}{lcccr}
\hline & \multicolumn{4}{c}{ Herd type $^{1}$} \\
\cline { 2 - 4 } $\begin{array}{l}\text { Gastrointestinal } \\
\text { parasite eggs }\end{array}$ & Organic & $\begin{array}{c}\text { Pasture access } \\
\text { conventional }\end{array}$ & $\begin{array}{c}\text { Indoor, } \\
\text { conventional }\end{array}$ & $P_{\text {-value }}^{2}$ \\
\hline Strongyle & $100^{\mathrm{a}}$ & $94^{\mathrm{a}}$ & $40^{\mathrm{b}}$ & $<0.01$ \\
Trichuris & $52^{\mathrm{b}}$ & $65^{\mathrm{b}}$ & $100^{\mathrm{a}}$ & 0.02 \\
Nematodirus & $42^{\mathrm{b}}$ & $82^{\mathrm{a}}$ & $20^{\mathrm{b}}$ & $<0.01$ \\
Moniezia & $70^{\mathrm{a}}$ & $12^{\mathrm{b}}$ & 0.01 \\
Dictyocaulus & 18 & 0 & 0 & 0.07 \\
Strongyloides & 15 & 12 & 20 & 0.84 \\
Capillaria & 15 & 12 & 0 & 0.42 \\
Buxtonella & 6 & 6 & 0 & 0.73 \\
\hline
\end{tabular}

${ }^{\mathrm{a}, \mathrm{b}}$ Percentages in the same row with different superscripts were significantly different by pairwise Fisher's exact tests $(\alpha=0.05)$.

${ }^{1}$ Organic $(\mathrm{n}=33)$; Pasture access $=$ conventional herds on summer pasture $(\mathrm{n}=13)$; Indoor $=$ conventional herds with heifers housed indoors during summer $(\mathrm{n}=10)$.

${ }^{2} P$-values are for differences in overall percentages among the 3 herd types, based on Fisher's exact test $(\alpha=$ $0.05)$.

Both ORG and SC farms focused their fly control predominantly on the lactating cow herd and preweaned calves, whereas most MC farms used a fly control measure on all age groups. Fewer than half of the ORG and
SC farms controlled flies for their dry cows $(P<0.09)$ or replacement heifers $(P=0.07$; Table 5$)$.

Most farms did not use any of the questionnaire options for fly control, with the exception of sticky tapes,

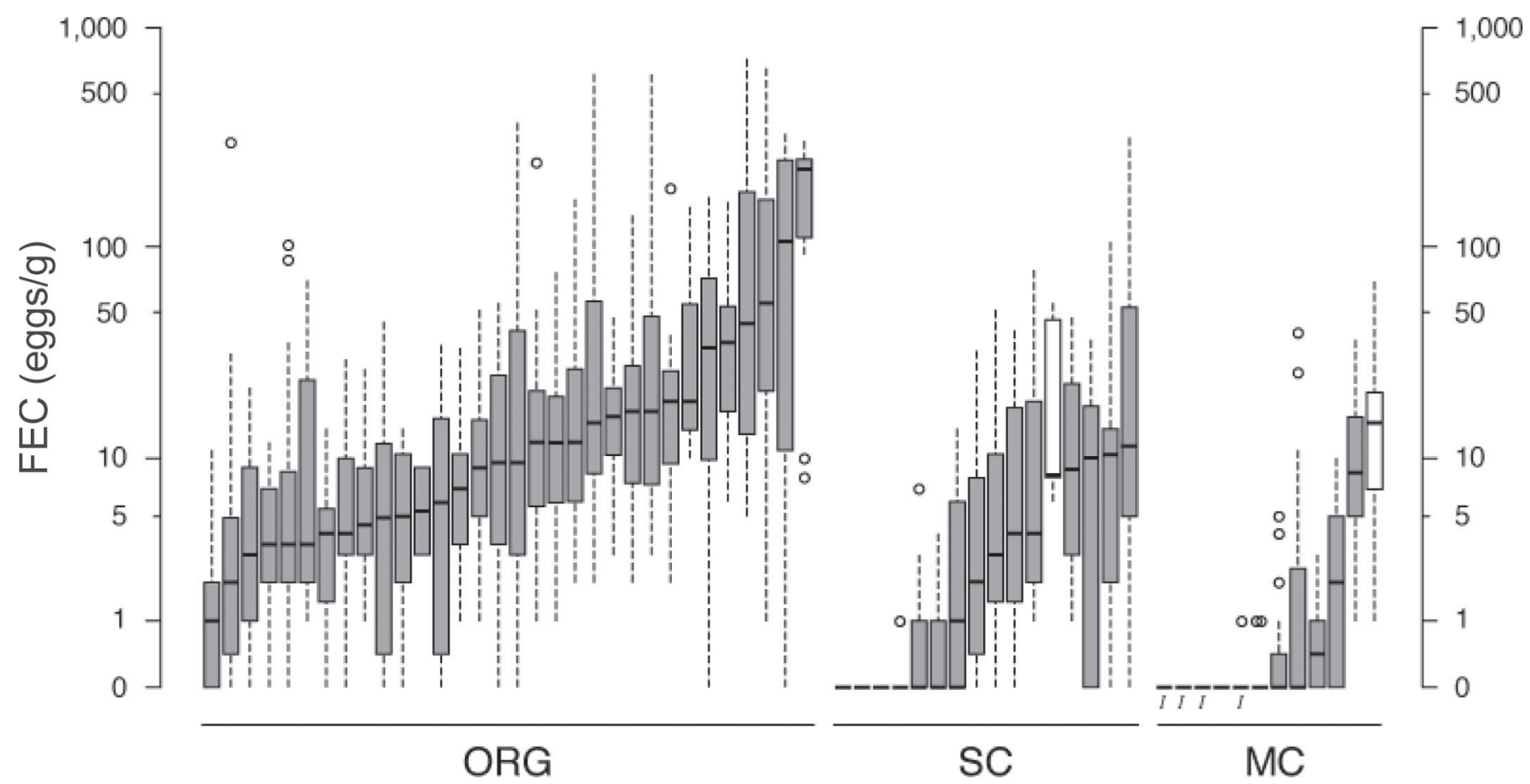

Figure 1. Ranked box-whisker plots of fecal egg counts (FEC) in eggs per gram [in $\log _{10}(x+1)$ scale] from individual heifers in 32 organic (ORG) dairies, 16 small conventional (SC) dairies, and 12 medium-sized conventional (MC) dairies in Minnesota, sampled once in June to November 2012. Median sample size was 20 heifers per dairy (range 2-21). Boxes are interquartile ranges $(r$ ), bold horizontal lines in boxes are medians, dashed lines are outward ranges within $\pm 1.5 r$ of median, and circles are outliers. One unfilled box among SC herds, 1 among MC, and $4 \mathrm{MC}$ herds marked with " $\Gamma$ " were from conventional herds housed continuously indoors; all remaining herds were on summer pasture. 
Table 5. Percentages of Minnesota dairies that reported use of different fly control measures in 2012 (multiple answers were possible)

\begin{tabular}{|c|c|c|c|c|}
\hline \multirow[b]{2}{*}{ Item } & \multicolumn{3}{|c|}{ Herd type $^{1}$} & \multirow[b]{2}{*}{$P$-value ${ }^{2}$} \\
\hline & ORG & $\mathrm{SC}$ & $\mathrm{MC}$ & \\
\hline Reported use of fly control of any kind & 89 & 60 & 92 & 0.04 \\
\hline \multicolumn{5}{|l|}{ Type of animals receiving fly control } \\
\hline Milking cows & 86 & 60 & 85 & 0.25 \\
\hline Dry cows & $34^{\mathrm{b}}$ & $43^{\mathrm{ab}}$ & $69^{\mathrm{a}}$ & 0.09 \\
\hline Replacement heifers & $25^{\mathrm{b}}$ & $43^{\mathrm{ab}}$ & $62^{\mathrm{a}}$ & 0.07 \\
\hline Calves & 57 & 50 & 77 & 0.34 \\
\hline \multicolumn{5}{|l|}{ Types of fly control measures used } \\
\hline Walk through traps & 3 & 0 & 0 & $>0.90$ \\
\hline Baited jugs & 20 & 0 & 0 & 0.08 \\
\hline Sticky tapes & $71^{\mathrm{a}}$ & $0^{\mathrm{b}}$ & $39^{\mathrm{c}}$ & $<0.01$ \\
\hline Parasitic wasps & 14 & 0 & 0 & 0.21 \\
\hline Other fly control approaches & 82 & 71 & 85 & 0.64 \\
\hline Fly spray on animals & 60 & 0 & 39 & \\
\hline Spray/fog barns & 0 & 47 & 23 & \\
\hline Pour On & 6 & 13 & 7 & \\
\hline Feed additive & 0 & 15 & 0 & \\
\hline Other fly traps & 6 & 0 & 0 & \\
\hline Chickens & 9 & 0 & 0 & \\
\hline Lime on cows & 3 & 0 & 0 & \\
\hline Rubbing device with dust & 3 & 0 & 15 & \\
\hline
\end{tabular}

${ }^{a-c}$ Percentages in the same row with different superscripts were significantly different by pairwise Fisher's exact tests $(\alpha=0.05)$.

${ }^{1} \mathrm{ORG}=$ organic herds $(\mathrm{n}=35) ; \mathrm{SC}=$ small conventional herds $(<200$ dry and milking cows, $\mathrm{n}=16)$; and $\mathrm{MC}$ $=$ medium-sized conventional herds $(\geq 200$ mature cows, $\mathrm{n}=12)$.

${ }^{2} P$-values are for differences in overall percentages among the 3 herd types, based on Fisher's exact test $(\alpha=$ $0.05)$.

which were used by most ORG herds and some MC herds. Interestingly, none of the SC herds used sticky tapes for fly control $(P<0.01)$. The most commonly used fly control tools were fly sprays on cattle, spraying or fogging of barns, and pour-on products. Only ORG producers used baited jugs $(n=7)$, parasitic wasps $(\mathrm{n}=6)$, chickens alongside the cows $(\mathrm{n}=3)$, or walkthrough traps $(\mathrm{n}=1)$ as fly control measures (Table 5$)$.

\section{Ectoparasites}

Excluding ringworm infections in heifers, we detected no difference among herd types in proportion of herds that reported seeing signs associated with lice or tailhead mange, such as excessive scratching and rubbing, hair loss or scabby skin conditions, in particular around the tail head, in their cattle during the winter of 20112012 (ORG: $48.6 \%$, SC: $57.1 \%$, MC: $53.9 \%$; $P=0.85$ ). Of those that saw such conditions, only $37.5,77.8$, and $71.4 \%$ of ORG, SC, and MC herds treated the animals with anything against lice or tail mange infections, respectively. Whereas SC herds and MC herds treated the skin conditions with pharmaceutical parasiticides, ORG herds did not use those products $(P<0.01)$. Instead, ORG producers used topical treatments such as brown sugar, diatomaceous earth, and iodine spray.

\section{DISCUSSION}

This survey is the first study to quantify the prevalence of different GI parasite eggs in dairy herds in the north-central region of the United States. Previous studies had focused on the description of the GI parasite burden or FEC in cow-calf herds in the upper Midwest (Stromberg and Corwin, 1993; Stromberg and Gasbarre, 2006; Hildreth et al., 2007). The few studies that had investigated GI parasites in dairy cows focused only on a specific gastrointestinal parasite such as Ostertagia (Sithole et al., 2005), did not distinguish between different helminth species (Hildreth et al., 2007), or considered only conventional herds that could use pharmaceutical dewormers (Sithole et al., 2005).

A strength of the present study is the diversity of dairy herd types studied and the large number of fecal samples analyzed. Furthermore, we attempted to collect a representative sample per herd (Gasbarre et al., 1996) and collected the samples during the transmission season (i.e., summer months). It was positive to note that the time of first turn out was comparable across herd types, if pasture access was given to animals. Additionally, the herds' production characteristics were comparable to other reports in the literature that compared organic and conventional herds (Zwald et al., 2004; Sato et al., 2005; Stiglbauer et al., 2013). 
However, there are also limitations to this study: the nominal goal of 20 fecal samples could not be obtained on all herds, the Wisconsin sugar float method does not allow for identification of Fasciola eggs, we sampled only once at each farm, and FEC may not necessarily reflect the actual parasite burdens of infected animals (Eysker and Ploeger, 2000). Furthermore, although most herds were visited in July and August, many ORG herds were visited slightly earlier than conventional herds, which could influence the results. Additionally, we did not record timing between the most recent deworming effort and sample collection date at each farm, and we did not characterize the general frequency of anthelmintic treatments at the different farms.

A surprising result of this study was that most ORG dairy farmers did not consider GI parasites to be a problem and did not consider parasite prevention when they set up their grazing schedule, even though they cannot routinely use dewormers. Furthermore, very few ORG dairy producers had dewormed heifers with ivermectin in the previous grazing season, or knew that fenbendazole and moxidectin had been added to the NOP. Considering that the former can only be done if a heavy infection had been diagnosed and if the organic certifier approves of its use, one might argue based on this observation alone that heifers in ORG dairy herds indeed might not have a high, clinically manifesting parasite infection.

Indeed, the average counts of various GI parasites were low overall, and were comparable between ORG and conventional herds. Very few individual ORG animals had total FEC >500 epg, which would be considered a heavy infection (Corwin, 1997), and the observed total FEC in conventional herds were similar to those described in mature cows of dairy herds in Sweden and the UK (Höglund et al., 2001; Fox et al., 2007; Maggs et al., 2008). In contrast, FEC on ORG dairies were lower than the previously described nematode FEC in conventional cow-calf herds in the upper Midwest (Hildreth et al., 2007) or from a recent study in Sweden (Areskog et al., 2013).

The fact that the average strongyle-type FEC was higher in the ORG herds than in conventional herds suggests that strongyles, which are generally most pathogenic nematodes of cattle, may be more problematic in ORG herds than in conventional herds. The higher strongyle FEC in ORG herds may be attributable to exposure on pasture, as was suggested by Sithole et al. (2005), who found higher Ostertagia FEC in dairy herds with pasture access than in herds with limited or no pasture access. Considering that ponds and streams - marshy areas in general - provide a good environment for survival of infective larvae, it was not surprising to see that the presence of a stream on pasture was associated with increased strongyle FEC in this study.

Exposure to parasites on pasture likely explains, too, why ORG herds were more likely than herds in confinement systems to have Moniezia or Dictyocaulus infections. The observed prevalence of Moniezia was comparable to those seen in cow-calf herds (Hildreth et al., 2007). Interestingly, all ORG herds that had only one type of parasite egg present had Moniezia, whereas all conventional herds with only one parasite had Trichuris. The latter was also more likely to be present in MC herds than ORG herds, suggesting that Trichuris infections are associated with animals housed indoors. However, most management practices were very much associated with herd type and more data are needed to identify the effect of various management practices on Trichuris infections.

Most farms of all 3 types did implement some kind of fly control measure, but SC farms were less likely to do so than ORG or MC farms. Furthermore, of farms that performed fly control, most emphasis was placed on the milking herd and preweaned calves; dry cows and heifers were less likely to be treated on ORG and SC farms. In contrast, a greater percentage of MC farms had fly control measures in place for all 4 cattle groups. One might speculate that this was because these 2 animal groups have the most day-to-day interaction with humans on smaller farms and that (1) flies would bother the human caretaker, (2) the discomfort of milking cows would be noticed due to kicks during milking and lost milk production, or (3) it was not seen as economically viable to spend money for fly treatment of replacement heifers and dry cows.

Approaches for fly control differed between ORG and conventional farms. Organic farmers were unlikely to spray or fog the barn with insecticides against flies, probably due to the lack of NOP fly control products. Instead, ORG managers were likely to use botanical fly sprays, parasitic wasps, or chickens for fly control. Chickens are used because they will forage for grain through manure patties on pasture and therefore spread them out. This will dry the patties quicker and impair the growth of fly and other larvae. Chickens were not used at all by conventional farms.

Compared with flies, other ectoparasites received even less attention on dairy farms. Although many herd managers saw lice or mange infestations, very few ORG farmers reported treating these infestations, whereas conventional farmers used various topical pharmaceuticals.

In conclusion, most ORG dairy farmers did not perceive GI parasites as a problem on their farm; and 
although ORG farms did have, on average, higher strongyle egg counts than conventional dairy farms, the parasite FEC were low overall across herd types. Lice or mange infestations were seen on approximately half of all herds and were not treated consistently. Similarly, fly control was practiced predominantly only for milking cows and preweaned heifers regardless of herd type.

\section{ACKNOWLEDGMENTS}

The authors thank the dairy producers for participating in this study and Jason Lombard (USDA-Animal and Plant Health Inspection Service, Fort Collins, CO) for access to the USDA NAHMS surveys. In addition, we thank Sue Prouty, Carla Barstow, and Giordana Costa (University of Minnesota) for their help in the laboratory and the Minnesota Agricultural Experiment Station for funding.

\section{REFERENCES}

AMS (Agricultural Marketing Service). 2012. Code of Federal Regulations. Title 7. Part 205: National Organic Program. Accessed Sep. 4, 2013. http://www.ecfr.gov/cgi-bin/text-idx?c=ecfr\&sid=3f34f4 c22f9aa8e6d9864cc2683cea02\&tpl=/ecfrbrowse/Title07/7cfr205_ main_02.tpl.

Areskog, M., B. L. Ljungsström, and J. Höglund. 2013. Limited efficacy of pour-on anthelmintic treatment of cattle under Swedish field conditions. Int. J. Parasitol. Drugs Drug Res. 3:129-134.

Bliss, D. H., and W. G. Kvasnicka. 1997. The fecal examination: A missing link in food animal practice. Compend. Contin. Educ. Pract. Vet. 19(Suppl. 4):S104-S109.

Corwin, R. M. 1997. Economics of gastrointestinal parasitism of cattle. Vet. Parasitol. 72:451-457.

ERS (Economic Research Service). 2013. Table 2. U.S. Certified organic farmland acreage, livestock numbers, and farm operations. Accessed Feb. 10, 2015. http://www.ers.usda.gov/data-products/ organic-production.aspx.

Eysker, M., and H. W. Ploeger. 2000. Value of present diagnostic methods for gastrointestinal nematode infections in ruminants. Parasitology 120:S109-119.
FARM. 2013. The National Dairy Farmers Assuring Responsible Management: Animal Care Reference Manual. National Milk Producers Federation, Arlington, VA.

Fox, M. T., M. Hutchinson, A. Riddle, and A. B. Forbes. 2007. Epidemiology of subclinical dairy cow nematode infections on five farms in England in 2002 and a comparison with results from 1978 to 1979. Vet. Parasitol. 146:294-301.

Gasbarre, L. C., E. A. Leighton, and D. Bryant. 1996. Reliability of a single fecal egg per gram determination as a measure of individual and herd values for trichostrongyle nematodes of cattle. Am. J. Vet. Res. 57:168-171.

Hildreth, M. B., W. B. Epperson, and K. J. Mertz. 2007. Effect of longitude and latitude on fecal egg and oocyst counts in cow-calf beef herds from the United States Northern Great Plains. Vet. Parasitol. 149:207-212.

Höglund, J., C. Svensson, and A. Hessle. 2001. A field survey on the status of internal parasites in calves on organic dairy farms in southwestern Sweden. Vet. Parasitol. 99:113-128.

Maggs, L. A., S. Athanasiadou, L. Sherwood, and M. J. Haskell. 2008. Levels of parasitism on organic and non-organic dairy farms in Scotland. Vet. Rec. 162:345-346.

R Core Team. 2014. R: A language and environment for statistical computing. R Foundation for Statistical Computing, Vienna, Austria.

Sato, K., P. Bartlett, R. Erskine, and J. Kaneene. 2005. A comparison of production and management between Wisconsin ORG and conventional dairy herds. Livest. Prod. Sci. 93:105-115.

Sithole, F., I. Dohoo, K. Leslie, L. DesCoteaux, S. Godden, J. Campbell, H. Stryhn, and J. Sanchez. 2005. Effect of eprinomectin treatment at calving on milk production in dairy herds with limited outdoor exposure. J. Dairy Sci. 88:929-937.

Stiglbauer, K. E., K. M. Cicconi-Hogan, R. Richert, Y. H. Schukken, P. L. Ruegg, and M. Gamroth. 2013. Assessment of herd management on organic and conventional dairy farms in the United States. J. Dairy Sci. 96:1290-1300.

Stromberg, B. E., and R. M. Corwin. 1993. Epizootiology of Ostertagia ostertagi in cow-calf production systems in the American Midwest. Vet. Parasitol. 46:297-302.

Stromberg, B. E., and L. C. Gasbarre. 2006. Gastrointestinal nematode control programs with an emphasis on cattle. Vet. Clin. North Am. Food Anim. Pract. 22:543-565.

Zwald, A. G., P. L. Ruegg, J. B. Kaneene, L. D. Warnick, S. J. Wells, C. Fossler, and L. W. Halbert. 2004. Management practices and reported antimicrobial usage on conventional and organic dairy farms. J. Dairy Sci. 87:191-201. 